\title{
SPACES OF CONTINUOUS FUNCTIONS
}

\section{S. B. MYERS}

Let $X$ be a completely regular topological space, $B(X)$ the Banach space of real-valued bounded continuous functions on $X$, with the usual norm $\|b\|=\sup _{x \in X}|b(x)|$. A subset $G \subset B(X)$ is called completely regular (c.r.) over $X$ if given any closed subset $K \subset X$ and point $x_{0} \in X-K$, there exists a $b \in G$ such that $b\left(x_{0}\right)=\|b\|$ and $\sup _{x \in K}|b(x)|<\|b\|$. A topological space $X$ is completely regular in the usual sense if and only if $B(X)$ is c.r. over $X$.

A Banach space $B$ is said to act completely regularly (c.r.) on $X$ if $B$ is equivalent to a closed linear subspace of $B(X)$ which is c.r. over $X$. It is known [6] $]^{1}$ that if $X$ is compact, ${ }^{2}$ a closed linear subspace of $B(X)$ c.r. over $X$ determines the topology of $X$. By this is meant that if $X_{1}$ and $X_{2}$ are compact, and a Banach space $B$ acts c.r. on both $X_{1}$ and $X_{2}$, then $X_{1}$ is homeomorphic to $X_{2}$. If $B$ acts c.r. on $X$ (compact or not), $X$ is homeomorphically imbeddable in the surface of the unit sphere in $B_{w}^{*}$, the conjugate space to $B$ under the weak-* topology, and for each $b \in B$ and $x \in X$ we have the formula $b(x)=\inf _{t \in T}[\|b+t\|-\|t\|]$, where $T=\{t \in B \mid t(x)=\|t\|\}$.

If we weaken the definition of complete regularity so that $G$ is c.r. over $X$ means that for every closed set $K \subset X$ and point $x_{0} \in X-K$ there is a $b \in G$ such that $b\left(x_{0}\right)=\|b\|, \sup _{x \in K} b(x)<\|b\|$, then a closed linear subspace of $B(X)$ c.r. over $X$ does not necessarily determine the topology of $X$. For example, if $X$ consists of just two points, $x_{1}$ and $x_{2}$, then the subspace $G$ of $B(X)$ consisting of all $b \in B(X)$ such that $b\left(x_{1}\right)=-b\left(x_{2}\right)$ is c.r. over $X$ according to the weakened definition, yet it is equivalent to the space $B(Y)$, where $Y$ consists of a single point.

Proper closed linear subspaces of $B(X)$ which are c.r. over $X$ exist in general for both compact and non-compact $X$, and may contain the constant functions. This is in contrast to the situation when $B(X)$ is made into a normed ring (Banach algebra) $R(X)$ or into a Banach lattice $L(X)$; if $X$ is compact, no proper closed subring of $R(X)$ containing the constant functions can be c.r. over $X[8]$, and no proper closed sublattice of $L(X)$ containing the constant functions can be c.r. over $X[4]$.

Since topological properties of $X$ must be reflected in metric and

Presented to the Society, February 28, 1948; received by the editors April 5, 1948.

${ }^{1}$ Numbers in brackets refer to the bibliography at the end of the paper.

2 "Compact" means bicompact and Hausdorff. 
algebraic properties of $B(X)$, and in such properties of every closed linear subspace $G$ of $B(X)$ which is c.r. over $X$, it would appear to be fruitful to investigate the possibilities of existence of a $G$ of a particularly simple sort, for example, separable, or finite-dimensional, or reflexive. Such a study is made here.

In the next few paragraphs necessary and sufficient conditions are proved that for a given Banach space $B$ there exists a compact $X$ such that $B$ acts c.r. on $X$. Later, we point out that $B(X)$ is itself rarely separable, finite-dimensional, or reflexive, and the existence of a c.r. subspace of $B(X)$ with such properties is investigated.

Let $B$ be any Banach space; by a $T$-set, we mean a maximal subset $T$ of $B$ with the property that for any finite subset $b_{1}, \cdots, b_{n}$ of $T,\left\|\sum b_{i}\right\|=\sum\left\|b_{i}\right\|$. Every $b \in B$ is contained in such a $T$-set.

Let $E_{w}^{*}$ be the solid unit sphere in $B_{w}^{*}$, and let $S_{w}^{*}$ be the surface of $E_{w}^{*}$. It is known that $E_{w}^{*}$ is compact [1].

THEOREM 1. Given a Banach space $B$, the following conditions are necessary and sufficient that there exist a compact $X$ such that $B$ acts c.r. on $X$ :

(1) For each $T$-set in $B$, there is a unique point $x_{T} \in S_{w}^{*}$ such that $x_{T}(t)=\|t\|$ for all $t \in T$.

(2) The set $M \subset S_{w}^{*}$ of all such $x_{T}$ is closed in $B_{v}^{*}$.

(3) $M$ is the union of two disjoint closed antipodal subsets $\Omega,-\Omega$.

Proof. The necessity of the conditions follows directly from [6, Theorem 4.1 and Lemma 2.3]. To prove the sufficiency, we show that hypotheses (1), (3) imply that $B$ acts c.r. on $\Omega$. Consider the linear mapping $C(B) \subset B(\Omega)$, which assigns to each $b \in B$ the function $b(x)$ defined by the formula $b(x)=x(b)$ for all $x \in \Omega$. It is clear that $C(0)$ is the function $\equiv 0$ over $\Omega$. Also, if $C(b)=C(0)$, then $b(x) \equiv 0$ over $\Omega$, hence over $M$, and in particular $b\left(x_{T}\right)=0$ where $x_{T}$ is the point in $M$ which corresponds by hypothesis (1) to the $T$-set containing $b$; hence $\|b\|=0$. It follows that $C$ is one-to-one. Since $M \subset S_{v o}^{*},-\|b\|$ $\leqq b(x) \leqq\|b\|$ for all $b \in B$ and all $x \in M$, and since $b\left(x_{T}\right)=\|b\|$ and $b\left(-x_{T}\right)=-\|b\|$, we see that $\|b\|=\sup _{x \in \Omega}|b(x)|$. Thus $C$ is normpreserving, hence an equivalence. Since $B$ is complete, $C(B)$ is complete and hence closed in $B(\Omega)$. It remains to show that $C(B)$ is c.r. over $\Omega$. Let $x_{0}$ be any point in $\Omega$, let $D$ be any closed subset of $\Omega$ not containing $x_{0}$. Let $K=D \cup-D ; K$ is closed in $M$. Let $T$ be the $T$-set in $B$ such that $x_{0}(t)=\|t\|$ for all $t \in T$, and let $\bar{K}$ be the closure of $K$ in $E_{w}^{*} ; \bar{K}$ does not contain $x_{0}$. For each $x \in \bar{K}$, there is a $t \in T$ such that $t(x)[=x(t)]<\|t\|$; for if $x$ is in $S_{w}^{*}$, this follows from (1), and if $\|x\|<1$ then $t(x)<\|\bar{t}\|$. Since $\bar{K}$ is compact, and $t(x)$ is a continuous 
function of $x$ for every $t \in B$, there is a finite set $t_{1}, \cdots, t_{n} \in T$ such that for each $x \in \bar{K}$, at least one $t_{i}$ has the property $t_{i}(x)<\left\|t_{i}\right\|$. Hence if we let $\bar{t}=\sum t_{i}$, $\sup _{x \in \bar{K}} \bar{t}(x)<\|\bar{t}\|$, in particular $\sup _{x \in K} \bar{t}(x)<\|\bar{t}\|$. Since $K=-K$, we have $\sup _{x \in K}|\bar{t}(x)|<\|\bar{t}\|$, hence $\sup _{x \in D}|\bar{t}(x)|$ $<\|\bar{t}\|$. Thus $B$ acts c.r. on $\Omega$. The compactness of $\Omega$ follows from (2), (3) and the compactness of $E_{w}^{*}$.

Condition (1) of Theorem 1 could be replaced by the equivalent condition that for each $T$-set in $B$ the functional $F_{T}(b)=\inf _{t \in T}$ $[\|b+t\|-\|t\|]$ be linear over $B$ (see [6]). Furthermore, when (1) is satisfied, $x_{T}=F_{T}$.

The following three results, the first two of which are stated without proof since they are known, indicate properties which $B(X)$ in general fails to have.

THEOREM 2. If $X$ is completely regular, $B(X)$ is separable if and only if $X$ is compact and metrizable [5].

THEOREM 3. If $X$ is completely regular, $B(X)$ is finite-dimensional if and only if $X$ consists of a finite number of points.

THEOREM 4. If $X$ is completely regular, $B(X)$ is reflexive if and only if $X$ consists of a finite number of points.

Proof. If $X$ has only a finite number of points, $B(X)$ is reflexive because it is finite-dimensional. Now suppose $X$ completely regular and infinite, and $B(X)$ reflexive. Let $\bar{X}$ be the Cech compactification of $X$. Then $B(\bar{X})$ is equivalent to $B(X)$, hence reflexive. Therefore the unit sphere $E$ in $B(\bar{X})$ is weakly compact. We obtain a contradiction by constructing a sequence in $E$ with no weak limit point. Let $\{x\}$ be an infinite sequence of distinct points of $\bar{X}$, and let $x$ be a limit point of $\{x\}$ not a member of $\{x\}$; let $b_{i}$ be an element of $B(\bar{X})$ with the property that $\left\|b_{i}\right\|=1, b_{i}(x)=1, b_{i}\left(x_{j}\right)=0$ for $j \leqq i ; b_{i}$ exists due to the complete regularity of $X$. If $b$ is a weak limit point of $\{b\}$, it follows that $b(x)=1, b\left(x_{j}\right)=0$ for $j=1,2, \cdots$, contradicting the fact that $b$ is continuous and $x$ is a limit point of $\{x\}$.

THEOREM 5. If $E$ is the unit sphere in any Hilbert (inner product) space $H$, there exists a closed linear reflexive subspace $L$ of $B(E)$ which is c.r. over $E$ and is isomorphic to $H$ if $H$ is of infinite dimension and $n+2$-dimensional if $H$ is $n$-dimensional.

Proof. Let $c, d$ be real numbers, let $h \in H$, let $P \in E$. For fixed $c, d, h$ the function $f_{c, d, h}(P)=c\|P\|^{2}+P \cdot h+d$ is a bounded continuous function over $E$. The set of all such functions as $c, d$ range over the real numbers and $h$ ranges over $H$, with $\left\|f_{c, d, h}\right\|=\sup _{P \in E}$ $\left|f_{c, d, h}(P)\right|$, forms a linear subspace $L$ of $B(E)$. 
Consider the mapping $C\left(H_{1} \times H_{1} \times H\right)=L$, where $\times$ denotes direct sum, $C(c, d, h)=f_{c, d, h}$, and $H_{1}$ is the euclidean line. $C$ is clearly linear and continuous. $C$ is also one-to-one, for if $C(c, d, h)=C(\bar{c}, \bar{d}, \bar{h})$ then $(c-\bar{c})\|P\|^{2}+P \cdot(h-\bar{h})+(d-\bar{d})=0$ for all $P \in E$; by taking $P=0$ we see that $d=\bar{d}$, and by taking $P=k(h-\bar{h})$, where $k>0$ is so small that $k(h-\bar{h})$ is in $E$ and $k \neq 1 /(\bar{c}-c)$, we see that $h=\bar{h}$, hence $c=\bar{c}$. To show that $C^{-1}$ is continuous, we must show that given $\epsilon>0$ there exists a $\delta$ such that if $\sup _{P \in E}\left|f_{c, d, h}(P)\right|<\delta$ then $\left(c^{2}+d^{2}+\|h\|^{2}\right)^{1 / 2}<\epsilon$. Using $\delta=\epsilon / 11^{1 / 2}$, by taking $P=0$ we get $|d|$ $<\epsilon / 11^{1 / 2}$. By taking $P=h /\|h\|$ we get $|c+\|h\|+d|<\epsilon / 11^{1 / 2}$, and by taking $P=-h /\|h\|$ we get $|c-\|h\|+d|<\epsilon / 11^{1 / 2}$, hence $\|h\|<\epsilon / 11^{1 / 2}$. Hence $c<3 \epsilon / 11^{1 / 2}$, and $\left(c^{2}+d^{2}+\|h\|^{2}\right)^{1 / 2}<\epsilon$.

Thus $C$ is an isomorphism between $H_{1} \times H_{1} \times H$ and $L$. But $H_{1} \times H_{1}$ $\times H$ is complete and reflexive, hence $L$ is complete (and so closed in $B(E)$ ) and reflexive. ${ }^{3}$

If $H$ is infinite-dimensional, $H$ is isomorphic to $H_{1} \times H_{1} \times H$, hence to $L$. If $H$ is $n$-dimensional, $H_{1} \times H_{1} \times H$ is $n+2$-dimensional, hence so is $L$.

To show $L$ is c.r. over $E$, let $P_{0} \in E$, and let $K$ be any closed set in $E-P_{0}$. Then by taking $c=-1 / 2, h=P_{0}, d=2$, we obtain $f_{c, d, h}(P)$ $=-\left(\left\|P-P_{0}\right\|^{2}\right) / 2+2+\left\|P_{0}\right\|^{2} / 2$, which is non-negative over $E$, and has the properties

$$
f_{c, d, h}\left(P_{0}\right)=\left\|f_{c, d, h}\right\| \sup _{P \in K}\left|f_{c, d, h}(P)\right|<f_{c, d, h}\left(P_{0}\right) .
$$

CoRollary. If $X$ is imbeddable in a Hilbert (inner product) space $H,{ }^{4}$ there exists a linear subspace of $B(X)$ which is c.r. over $X$, and which is the linear continuous image of $H_{1} \times H_{1} \times H$ (direct sum).

If $X$ is imbeddable in $H$ it is imbeddable in the solid unit sphere $E$ in $H$. Let $p(X) \subset E$ be this imbedding. The mapping $F[B(E)]$ $\subset B[p(X)]$, obtained by cutting down to $p(X)$ each continuous function on $E$, is clearly continuous and linear. If $L$ is the linear subspace of $B(E)$ furnished by Theorem 5 , then $F(L)$ is a linear subspace of

${ }^{3}$ A Banach space $\bar{B}$ isomorphic to a reflexive Banach space $B$ is reflexive. For let $I$ be such an isomorphism of $\bar{B}$ onto $B$, and let $I^{*}$ be the induced isomorphism of $B^{*}$ onto $\bar{B}^{*}$ defined by $\left[I^{*}(x)\right](\vec{b})=x[I(\bar{b})]$, where $x \in B^{*}$. Then if $\bar{F}$ is a continuous linear functional on $\bar{B}^{*}$, we see that $\bar{F}(\bar{x})=\bar{x}\left[I^{-1}(b)\right]$ for all $\bar{x} \in \bar{B}^{*}$, where $b$ is the point in $B$ such that $\bar{F}\left[I^{*}(x)\right]=x(b)$ for all $x \in B^{*}$ (b exists because $B$ is reflexive). Hence $\bar{B}$ is reflexive.

${ }^{4} X$ is imbeddable in a Hilbert space if and only if it is metrizable; this follows by combining recent results of C. H. Dowker (Duke Math. J. vol. 14 (1947) pp. 639645) and A. H. Stone (Bull. Amer. Math. Soc. vol. 54 (1948) pp. 977-982). 
$B[p(X)]$ and clearly acts c.r. on $X$ and is the linear continuous image of $L$, hence of $H_{1} \times H_{1} \times H$.

THEOREM 6. $X$ is a finite-dimensional separable metric space if and only if there exists a finite-dimensional linear subspace of $B(X)$ which is c.r. over $X$.

Assume $X$ is finite-dimensional separable metric, and let $n$ be the smallest dimension of any euclidean space $E^{n}$ in which $X$ is homeomorphically imbeddable. Then $X$ is imbeddable in the solid unit sphere $E$ in $E^{n}$; let $p(X) \subset E$ be such an imbedding. Either $p(X)$ lies on no spherical surface in $E$, or else $p(X)$ (and hence $X$ ) is an $(n-1)$ sphere; for if $p(X)$ is a proper subset of an $(n-1)$-sphere it is contained in the manifold obtained by removing a point from the $(n-1)$ sphere, which is homeomorphic to $E^{n-1}$, contradicting the definition of $n$.

Suppose $p(X)$ lies on no spherical surface in $E$. Let $F(L)$ be the linear continuous image of the linear subspace $L$ of $B(E)$ (furnished by Theorem 5) under the mapping $F[B(E)] C B[p(X)]$ obtained by cutting down to $p(X)$ each continuous function on $E$. $F(L)$ is equivalent to a linear subspace of $B(X)$, and acts c.r. on $X$. Now $L$ is the space consisting of all functions on $E$ of the form

$$
f_{c, d, \bar{y}}(y)=c \sum_{i=1}^{n} y_{i}^{2}+\sum_{i=1}^{n} y_{i} \bar{y}_{i}+d
$$

where $\bar{y}=\left(\bar{y}_{1}, \cdots, \bar{y}_{n}\right)$ ranges over $E^{n}$ and $(y)=\left(y_{1}, \cdots, y_{n}\right)$ ranges over $E$. $L$ is closed in $B(E)$, and is isomorphic to $E^{n+2}$. The mapping $F$ is 1 -1 over $L$; for if $f_{c, d, \bar{y}}=f_{c^{\prime}, d^{\prime}, \bar{y}^{\prime}}$ over $p(X)$, then $f_{c-c^{\prime}, d-d^{\prime}, \bar{y}-\bar{y}^{\prime}}(y)=0$ for $y \in p(X)$, which by (1) means that $p(X)$ lies on an $(n-1)$-sphere or an $(n-1)$-plane in $E^{n}$, contradicting hypotheses. Hence $F(L)$ is the linear 1-1 continuous image of $E^{n+2}$, hence isomorphic to $E^{n+2}$.

If $p(X)$ is an $(n-1)$-sphere in $E$, it is clear that (unless $X$ consists of one or two points, in which case it is clear from the start that $B(X)$ is finite-dimensional) by deformation of $p(X)$ there is an imbedding $g(X)$ of $X$ into $E$ such that $q(X)$ lies on no sphere, which reduces the problem to the case just treated.

Now assume there is an $n$-dimensional linear subspace $M$ of $B(X)$ which is c.r. over $X$. A consequence of the complete regularity of $M$ over $X$ is that given $x, \bar{x} \in X$ there is a continuous function $b \in M$ such that $b(x) \neq b(\bar{x})$. Since $M$ is $n$-dimensional, we can write $b(x)$ $=\sum a_{i} b_{i}(x)$ where $b_{1}(x), \cdots, b_{n}(x)$ is a base in $M$ and, for at least one value of $i, b_{i}(x) \neq b_{i}(\bar{x})$. Then the mapping $f(X) \subset E^{n}$ which assigns to each $x \in X$ the point in $E^{n}$ with coordinates $b_{1}(x), \cdots, b_{n}(x)$ 
is one-to-one. It is clearly continuous. Also $f^{-1}$ is continuous. For let $x_{0} \in X$, and let $O\left(x_{0}\right)$ be any open set containing $x_{0}$. Since $M$ is c.r. over $X$, there is a $b \in M$ and a $\delta>0$ such that $b\left(x_{0}\right)-b(x)>\delta$ for all $x \in X-O\left(x_{0}\right)$. Suppose $b=\sum a_{i} b_{i}$, and let $a=\max \left|a_{i}\right|$. Then for all $x$ such that $\left|b_{i}(x)-b_{i}\left(x_{0}\right)\right|<\delta / a n$ we have $\left|b\left(x_{0}\right)-b(x)\right|<\delta$, hence $x \in O\left(x_{0}\right)$. Thus $X$ is imbeddable in $E^{n}$, so $X$ is finite-dimensional separable metric.

TheOREM 7. If $X$ is completely regular, $B(X)$ contains a separable linear subspace c.r. over $X$ if and only if $X$ is separable metric.

First assume $X$ is separable metric. Then $X$ is imbeddable in ordinary Hilbert space $H$. By the corollary to Theorem 5 , there is a linear subspace $L$ of $B(X)$ which is c.r. over $X$ and is the linear continuous image of $H$. Since the continuous image of a separable space is separable, $L$ is separable.

Now assume $X$ is completely regular, and $B(X)$ contains a subspace $M$ both separable and c.r. over $X$. The unit sphere $E_{w}^{*}$ in $M_{w}^{*}$ contains a subset $X_{1}$ homeomorphic to $X$. The points of $E_{w}^{*}$ form an equicontinuous set of linear functionals on $M$. According to known results (see [7, Lemma 3.2 and Theorem 4.1]), the weak-* topology on $E^{*}$ and the compact-open topology on $E^{*}$ as a set of mappings of $M$ into the real line are identical, and the closure of $X_{1}$ in $E_{w}^{*}$ is compact metric. Hence $X_{1}$ is separable metric, and so is $X$.

\section{BIBLIOGRAPHY}

1. L. Alaoglu, Weak topologies of normed linear spaces, Ann. of Math. vol. 41 (1940) pp. 252-267.

2. S. Banach, Thêorie des opérations linéaires, Warsaw, 1932.

3. I. Gelfand and G. Silov, Über verschiedene Methoden der Einfïhrung der Topologie in die Menge der maximalen Ideale eines normierten Ringes, Rec. Math. (Mat. Sbornik) N.S. vol. 9 (1941) pp. 25-38.

4. S. Kakutani, Concrete representation of abstract (M)-spaces, Ann. of Math. vol. 42 (1941) pp. 994-1024.

5. M. and S. Krein, On an inner characteristic of the set of all continuous functions defined on a bicompact Hausdorff space, C. R. Acad. Sci. URSS. vol. 27 (1940) pp. 427430.

6. S. B. Myers, Banach spaces of continuous functions, Ann. of Math. vol. 49 (1948) pp. 132-140. 502.

7. ——, Equicontinuous sets of mappings, Ann. of Math. vol. 47 (1946) pp. 496-

8. M. H. Stone, Applications of the theory of Boolean rings to general topology, Trans. Amer. Math. Soc. vol. 41 (1937) pp. 375-481.

UNIVERSITY OF MichigaN 